\title{
Physical Motion of Mass Spring System Using MATLAB
}

\author{
Mahabad Abdulla \\ Department of Information Technology Erbil Technology Institute , Erbil Polytechnic University \\ .Mhabad Street 64, Erbil Iraq
}

\section{The research is financed by Mahabad Abdulla Sultan}

\section{Abstract}

This research explains and shows how widely MatLab can be used to provide a clear system that explains Mass Spring Vibrating Motion. Showing how it can be helpful in providing a visual explanation for mathematical problems that deals with a large number of data. The mentioned information will provides explanation of the rules of mass spring motion and how MatLab can be applied to create an actual motion that shows a similar effect of the mass on the shape of the spring by applying different weight of the mass and the force of spring.

Keywords: Damping Force, Gravitational Force Spring Force, Simulation, Loop

DOI: $10.7176 /$ RHSS/9-20-04

Publication date:October $31^{\text {st }} 2019$

\section{Introduction}

Mass Spring System is most widely used example of Simple Harmonic Motion (SHM). It has been widely experimented and mechanical behavior of the system is well defined in elementary texts of physics. Most of the work present is theoretical (Triana \& Fajardo, 2011). In addition to this several generalizations are made regarding spring mass correction and the analysis has been performed on oscillatory phenomenon in many cases such as mass on the spring.(Triana \& Fajardo, 2011)

Vibrating Systems are free from damping ideally. But, practically vibrations are damped. Mass Spring Damper models has number of applications(Chandra, 2018) which includes control parameters estimation, robotic control , control of vibrations.(Asadi-zeydabadi, 2014; Li \& Horowitz, 2001)There are number of factors responsible for damped forces. Among them are damping due to Coulomb friction, dry friction. A force which operates on relative change rate of displacement is known as damping force and it is opposite to spring.(Mushtaq, Ali, Anjam, \& Nazir, 2014).

Mass Spring Systems are widely used to build the mathematical model while using dynamics, In these systems the spring is connected with the mass.

Using Newton Second law of motion, this is given by

$$
F=m \frac{d x^{n}}{d t}
$$

And we know that Spring Force Relation

$$
F=-k x
$$

Substituting the spring force Relation

$$
-k x=m \frac{d x^{n}}{d t}
$$

The simple harmonic oscillation can be expresses in similar form. The common occurrence of spring is in form of metal coil. The idealization of spring is common. The displacement along spring axis is encountered by forces which force spring to its starting position. The idealization of spring depicts the simple behavior. Spring pushing will result in compressed spring and the string which is stretched is required to be pulled back (Li \& Horowitz, 2001).

The equation for damping force is given by

$$
F_{d}=-c \frac{d x}{d t}
$$

Here ' $c$ ' is the damping constant. In the mass spring mechanical system the damping force is always opposite to applied force and is proportional to velocity.(Becedas \& Mamani, 2007). The mass spring damper scheme is shown in figure 1(Becedas \& Mamani, 2007)

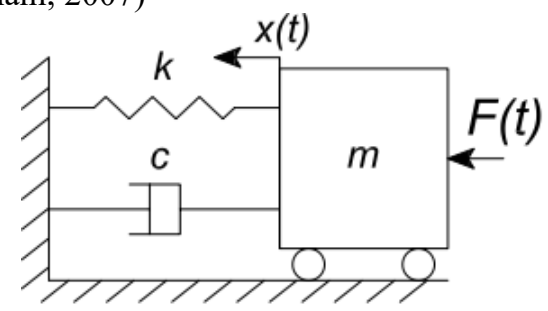

Figure 1 Mass Spring System 
The equation of model is Ordinary differential equation of second order but its homogenous model can be realized using Matlab and Simulink in which systems are represented in form of blocks. In Simulink the block for differentiating, integrating purpose are present and they perform predefined operations having one input and output. The output could be sent forward or feedback using certain interconnections. The Simulink can be used to model the dynamic system having independent variable time(Maddali, 2012).

\section{Theoretical Framework}

\subsection{Mass Spring System Composition Theory}

In the mass spring system, the dynamics of spring generate the internal forces while considering the stiffness the damping of spring. In this system, springs are used to connect mass points. The new position of mass and acceleration is determined using point dynamics

According to Newton Second Law of motion,

$$
F(t)=m \frac{d x^{n}}{d t}
$$

Calculating the displacement while deviating from initial position measured downward and is represented by $x(t)$. The sum of forces is acting on mass, which include

- Force in Downward Direction (Gravitational Force).

- Force in upward or Downward Direction either (Damping force)(Mushtaq et al., 2014)

- External Force either in upward or Downward Direction(Mushtaq et al., 2014)

- Force in upward or Downward Direction either (Spring force).

Now, neglecting the gravitational force and considering other forces

\section{Spring Force}

The force which obeys Hook's law is known as Spring Force Considering, when force is acted upward and spring is stretched

This happens when $\mathrm{x}>0$

$$
F_{s}=-k\left(s_{0}+x\right)
$$

$$
F=-K s_{0}+k(-x)=-k\left(s_{0}+x\right)
$$

Second Case, when force is acted downward and spring is compressed

This happens when $\mathrm{x}<0$

It can be seen that in both cases

\section{Damping Force}

$$
F_{s}=-k\left(s_{0}+x\right)
$$

The force opposite in direction to mass is known as Damping Force.(Gundes, 2014). The air resistance, dissipation energy, spring performance, resistive force due to mechanical effect may result in Damping Force and this force is proportional to rate of change of velocity.

Case 1: Upward Damping Force Action

When $\dot{x}>0$

$$
F_{d}=c \frac{d x}{d t}
$$

The mass would move downward and resulting displacement is increased

Case 1: Downward Damping Force Action When ' $x<0$

$$
F_{d}=-c \frac{d x}{d t}
$$

The mass would move downward and resulting displacement is increased. In both above mentioned cases $\mathrm{c}>0$

\subsection{Motion of Mass Spring System}

The mass placed between two strings is subjected to spring forces from both directions. Assuming the spring in rest position, when mass is displaced left the spring is compressed on left and other spring on the right is extended. As a result there would be restoring force on right from both springs

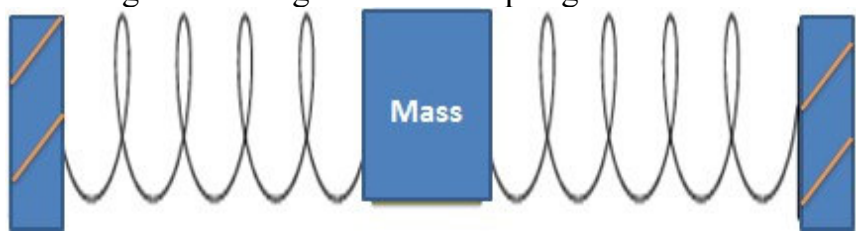

Figure 2 Block Diagram of Mass between two Springs 
And we know that Spring Force Relation

$$
F=-k x
$$

Now, if the mass is displaced a distance $\mathrm{x}$ to right also result in restoring force. As the spring on right is extended .The force on the right Spring is given by

$$
F_{\text {right spring }}=-k x
$$

The force exerted on the spring from left direction, mass will be pushed.

$$
F_{\text {left spring }}=-k x
$$

The basic framework was implemented using MATLAB and physical motion of two springs was analyzed which is given in following flow chart.

The sum of forces can be expressed as

$$
F_{\text {net }}=-2 k x
$$

The acceleration of mass is given by from Newton Second Law of Motion.

$$
\begin{gathered}
F_{\text {net }}=-2 k x=m a \\
a=\frac{-2 k}{m} x
\end{gathered}
$$

Previous researches mainly deals with theoretical and numerical evaluation of mass spring system but, in this research the mass spring moving between two springs is realized using MATLAB, their velocity and acceleration change with changing mass is observed and in the end different damping responses has been plotted and results are compared.

\section{Simulation and Results}

The physical motion of Mass Spring System is realized using Matlab. The code was written and for different values of Mass the Physical motion was examined. Here, in this case the weight of object that is linked to two springs is taken as input from user and can be varied. K constant of spring is kept constant.

\section{Physical Motion of Mass}

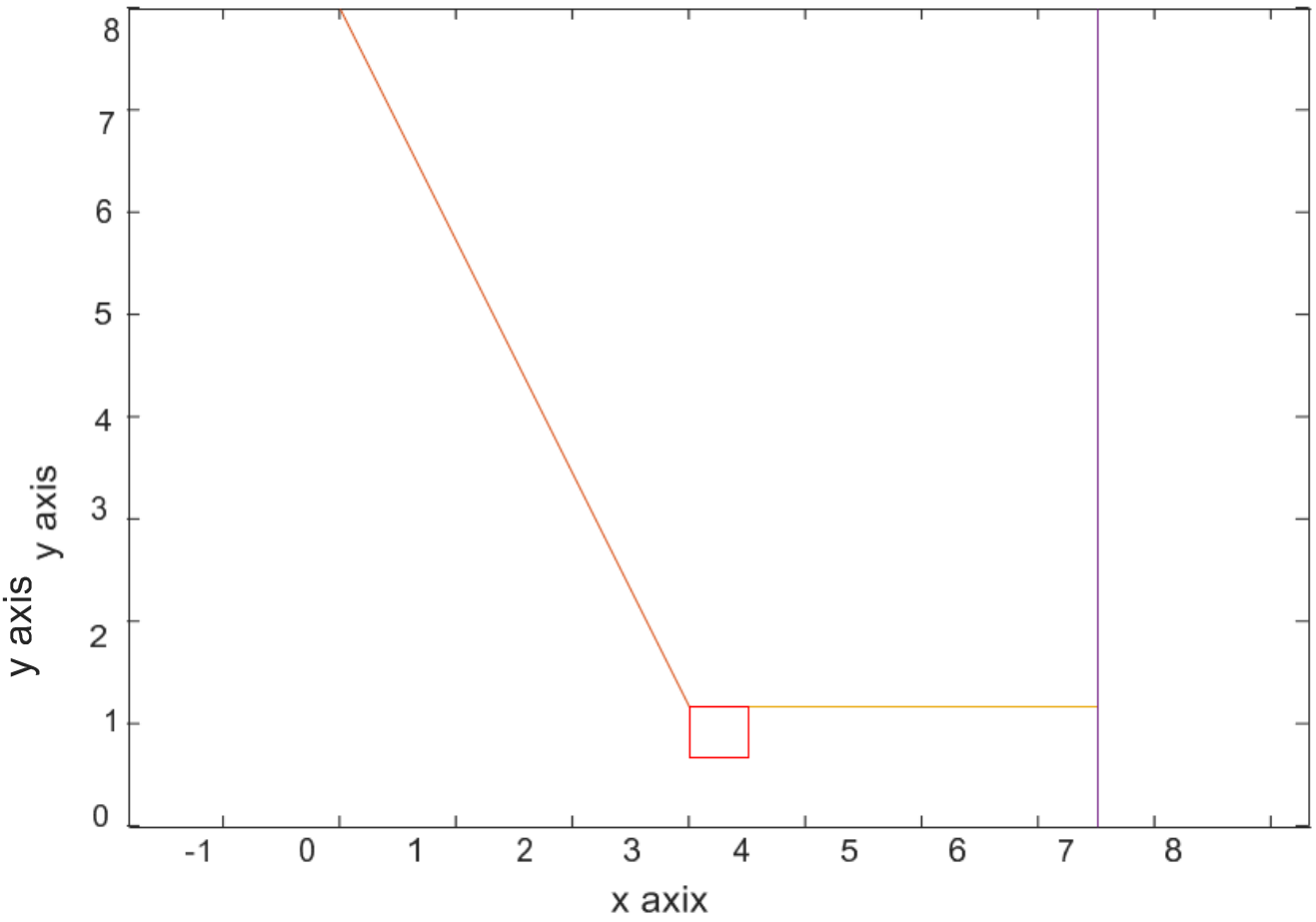

Figure 3 Physical Motion of Mass when $\mathbf{m = 1 0 0}$

The points of the line on the structure on $\mathrm{x}$ axis and $\mathrm{y}$ axis are given. The angular Velocity was calculated using formula 


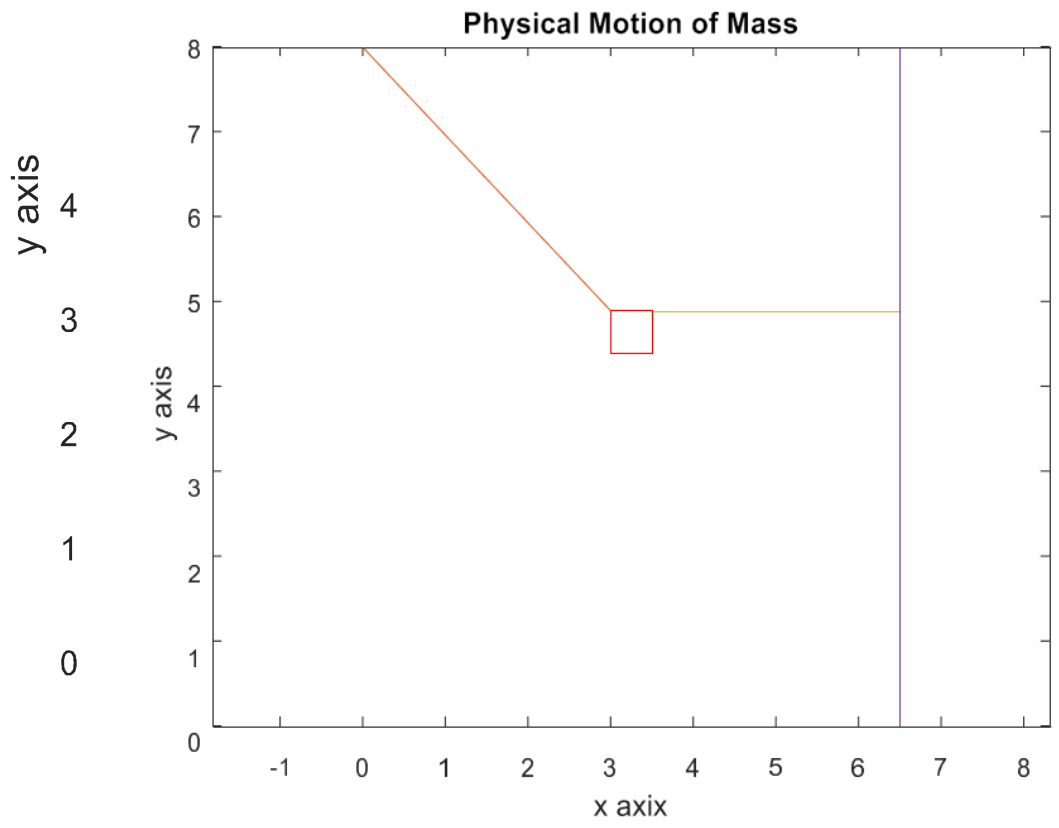

Figure 4 : Physical Motion of Mass when $\mathbf{m = 1 0}$

$$
w=\sqrt{\frac{K}{m}}
$$

The loop is created which then runs for evaluating the change in with respect to time. The changes in delta $y$ are computed from below equation

$$
\Delta y=A * \cos (w t(i)+\theta)
$$

The basic framework of this dynamic behavior realization Mass Spring Mechanical System is given in Figure 5. The simulation results for mass taken as 10 and then 100 is given in Figure 3 and figure 4 . The pause function is used to pause the movement of Spring after $0.1 \mathrm{~s}$. The mass is moved by adding values in the y axis of mass.

The basic framework of this dynamic behavior realization Mass Spring Mechanical System is given in Figure 5. The simulation results for mass taken as 10 and then 100 is given in Figure 3 and figure 4. The pause function is used to pause the movement of Spring after 0.1s. The mass is moved by adding values in the y axis of mass.

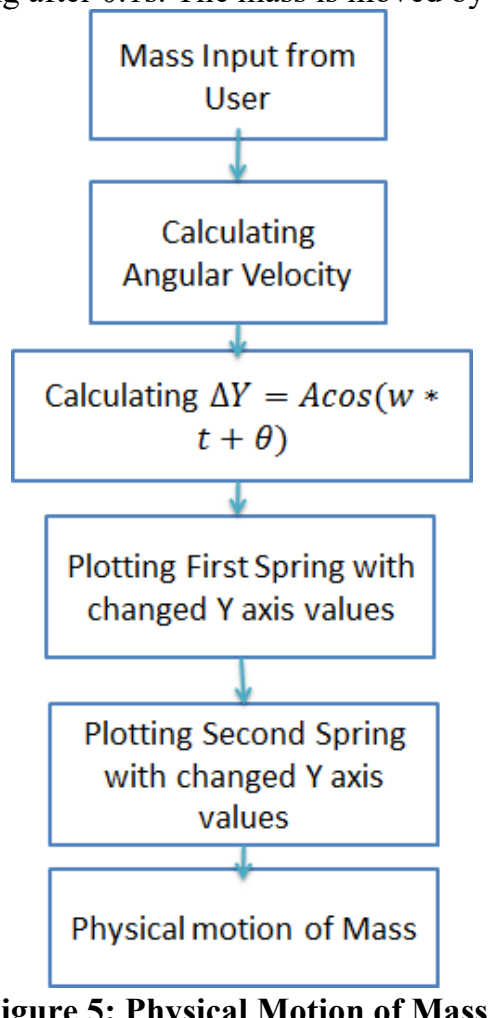


Mass moving between two springs was realized. The following figure represents the block diagram of Mass between two spring. The angular Velocity in this case as calculated using formula

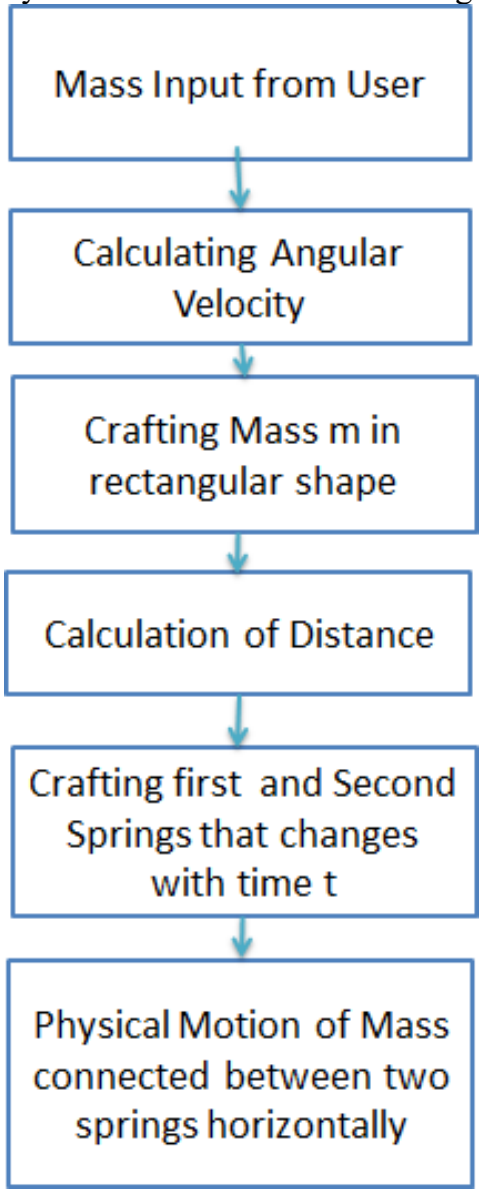

Figure 6 Basic Framework of Mass Moving between two Springs horizontally

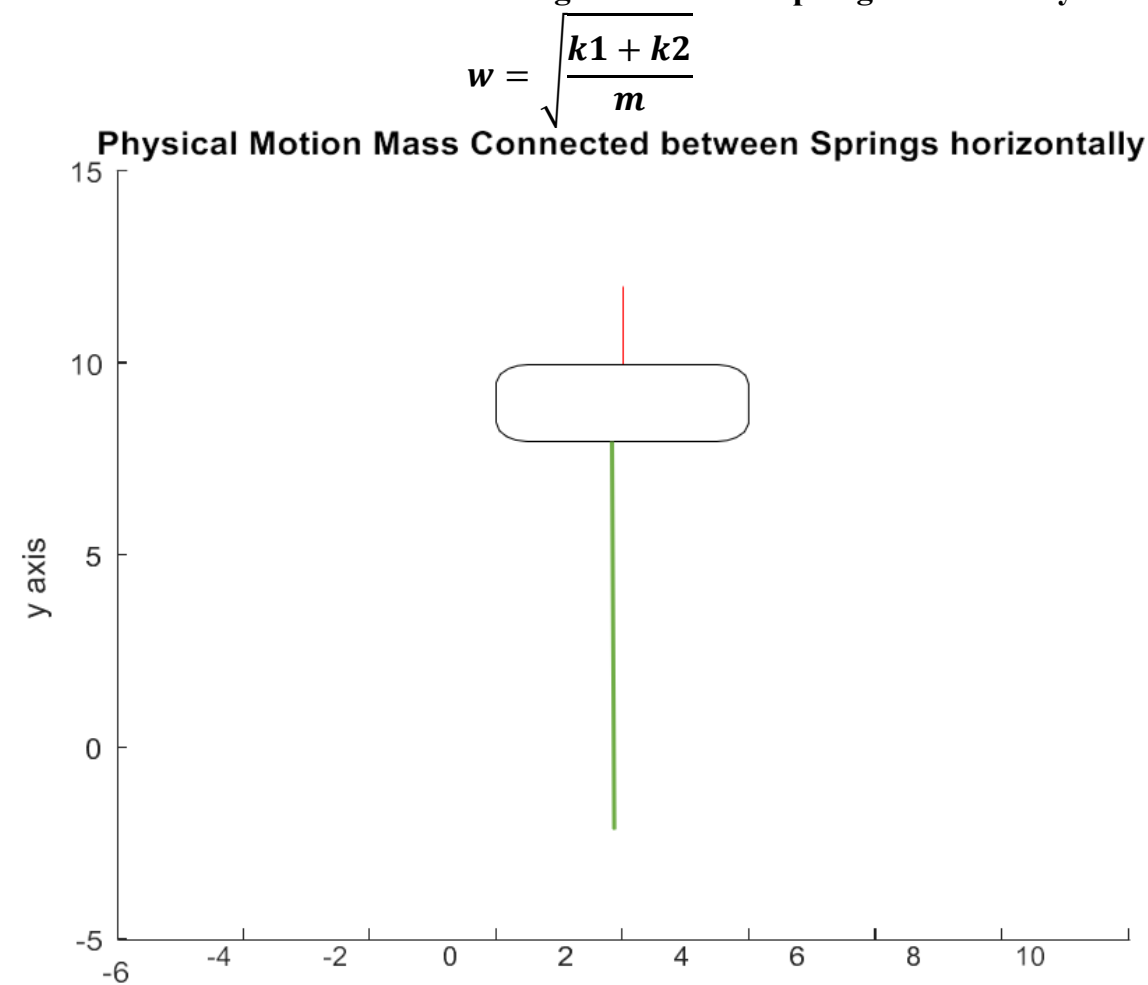

Figure 7 : Physical Motion of Mass between two springs when $\mathrm{m}=100$ 
The two colored lines show the spring. One is extended and other is compressed. The force on the Spring is given by

$$
F_{\text {spring }}=-k x
$$

In the above case value of mass is 100 and the compression and extension of spring was observed and angular velocity was calculated for this value which was found to be 3.763

In the next algorithm two masses are connected to two springs horizontally. The force was applied to the system. The total force experienced by first mass and second mass was calculated. Then acceleration of two masses was then calculated. The force experienced by the both masses on the spring was noted while changing different values of mass. The spring constant of two springs was taken as an input. The final velocity and then average velocity of two masses was realized using MATLAB. In the end new location of mass
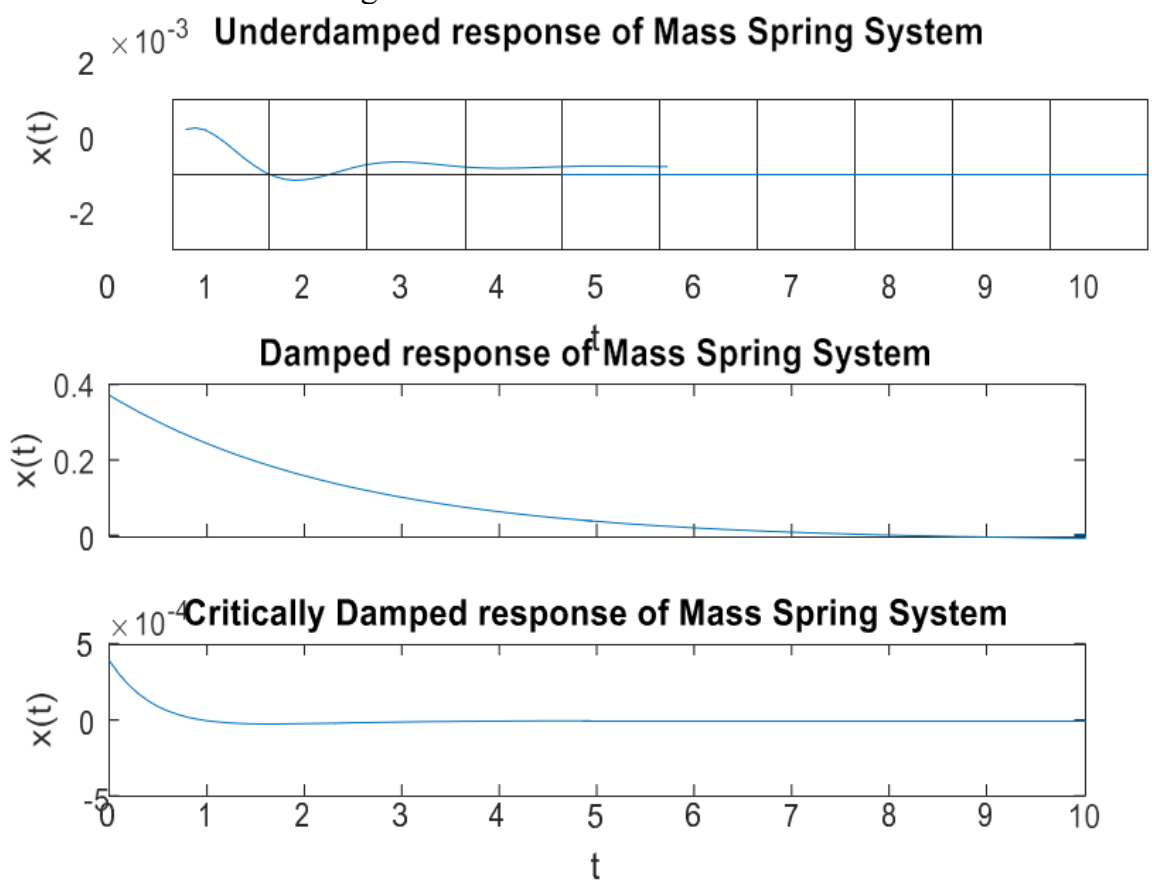

Figure 8 Different Damping Responses

The different damping responses was realized by changing the value of zeta and by using subplot command in MATLAB, their responses were compared and analyzed.

As observed from above graph in critically damped mass spring system, there are no oscillations. There is fastest return to time zero due to value of damping without oscillation(Garcia, 2015)

In case of under damped system damping ratio is between 0 and 1 . This is only and common cases in which vibrations are produced. In case of exponential decay, under damped systems does not produce oscillations. The systems available naturally vibrate in this manner(Garcia, 2015; J.Inman, 2014)

While in case of over damped system, the system does not oscillate and reaches exponentially to its rest position. The displacement of mass increases firstly, and then reaches its peak value

.After reaching its maximum value it comes back exponentially to its initial rest position which is zero. This response is slower as compared to critically damped system. The over damped system damping ratio is always greater than one(J.Inman, 2014)

\section{Conclusion}

The author in this research aims to physically realize the motion of mass spring system. Numerical solutions of mass spring system were evaluated using MATLAB .Various graphs have been plotted in MATLAB to determine the motion of single mass between two springs and two masses attached horizontally between two masses. Their velocity and acceleration were obtained in when different masses are applied. The plots give direct insight related to dynamic Mass Spring system and their different damping responses.

The results were similar to second order differential equations. While solving the second order equation gives a function which produces waves which are sine and cosine. The results obtained from the developed algorithm were same. This approach can be further extended for better visual understanding of different systems related to chemical, biological and other fields. 


\section{References}

Asadi-zeydabadi, M. (2014). Bessel Function and Damped Simple Harmonic Motion, (March), $26-34$.

Becedas, J., \& Mamani, G. (2007). Algebraic Identification Method for Mass-Spring-Damper System, (June 2014). Chandra, M. (2018). Spring Pendulum : A Nonlinear Paradigm, 1-20.

Garcia, M. (2015). GUI Matlab code to display damped, undamped, forced and unforced mass spring systems.

Gundes, P. (2014). Vibration of single degree of freedom systems y. J.Inman, D. (2014). Engineering-Vibrations2nd-Edition.pdf.

Li, Y., \& Horowitz, R. (2001). Active Suspension Vibration Control with Dual Stage Actuators in Hard Disk Drives. Maddali, R. K. (2012). MODELING ORDINARY DIFFERENTIAL EQUATIONS IN MATLAB SIMULINK ${ }^{\circledR}$. Mushtaq, M., Ali, A., Anjam, Y. N., \& Nazir, S. (2014). MODELING DAMPED MASS- SPRING SYSTEM IN MATLAB SIMULINK $®, 21(2), 21-28$.

Triana, C. A., \& Fajardo, F. (2011). The influence of spring length on the physical parameters of simple harmonic motion, 219. https://doi.org/10.1088/0143-0807/33/1/019 